\title{
Movement workshop
}

\author{
L.S. Cuijpers ${ }^{1,2}$ \\ ${ }^{1}$ Centre for Human Movement Sciences, UMCG, Groningen, The Netherlands \\ ${ }^{2}$ Heymans Institute for Psychological Research, University of Groningen, Groningen, the \\ Netherlands.
}

One of my favourite courses during my Movement Sciences education was "Motor Development and Learning". I was and still am fascinated by our ability to learn. Coming into the world as a helpless baby, confronted with ground reaction force and no means of moving yourself voluntary, we start to explore the dynamics of our bodies, always driven by curiousity and a will to reach for more. Striving to see more of the world and to become able to manipulate our environment.

We learn through action, exploring the dynamics of our bodies, experiencing the effects of our actions onto the world. Most of our students are inspired by their own experiences of moving themselves (e.g., in sports) and want to know more about human movement. Yet, in most of our classes, we limit learning and research to reading and discussing articles about movement and maybe watching how other people (struggle to) move.

This year, I developed a "Movement Workshop" for the 2nd year students partaking in the Motor Development and Learning course. In this workshop, students experienced theoretical concepts about motor development and learning by moving themselves. Inspired by Kinesica Movement Education, various movement games and puzzles challenged the students to find different movement solutions and coordination patterns.

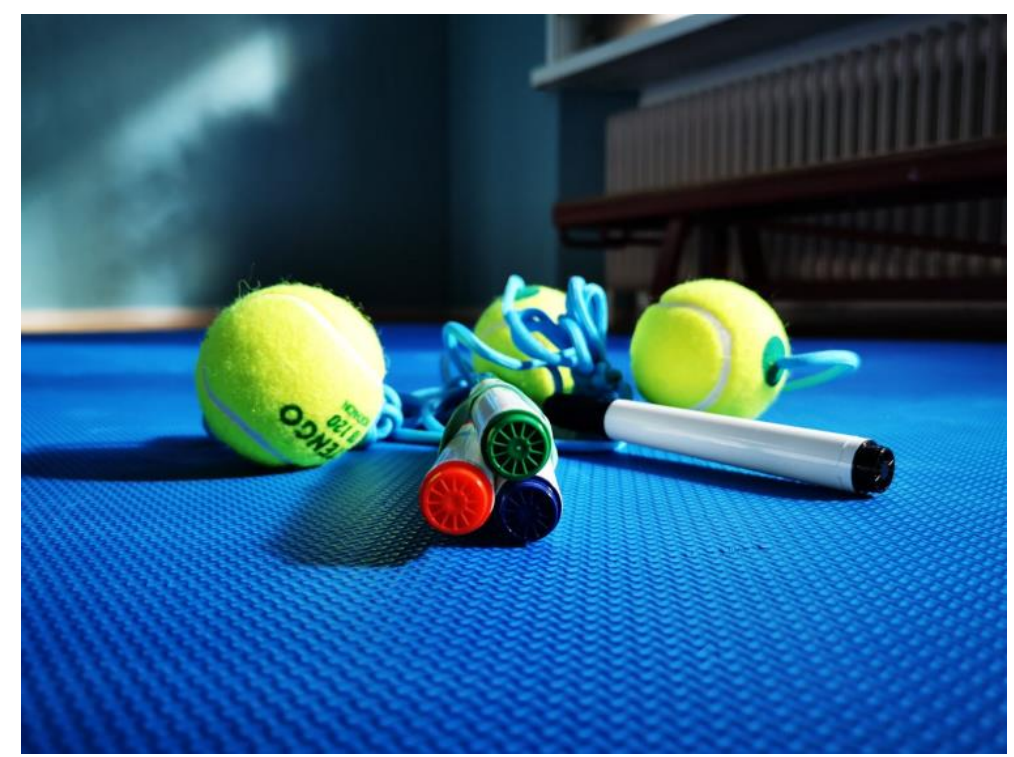

Toys and tools for teaching the movement workshop.

We started with a bodyscan in which students noticed where they could feel their heartbeat and some experiments on proprioception. Are you able to let your arms touch the ground at exactly the same time, or do you notice a difference between right and left? Are you able to let your index fingers touch in front of you if you keep your eyes closed? How does movement frequency affect these observations? 


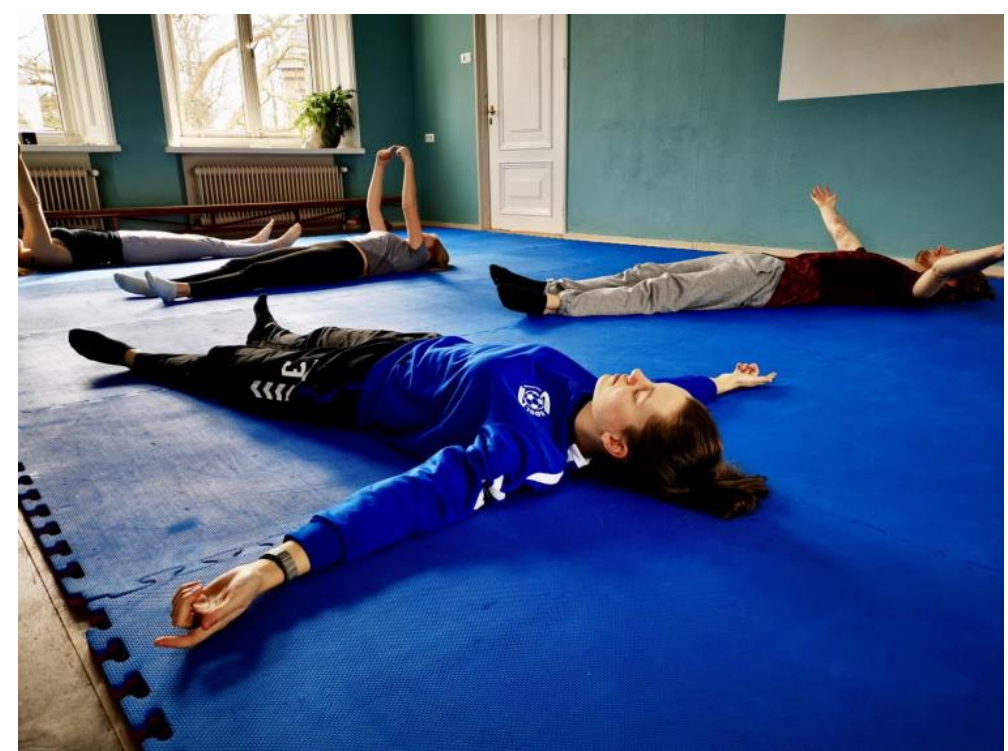

Experiment on proprioception. Can you let your fingers touch in front of you while keeping your eyes closed?

Next, we discussed various reflexes, such as the grasping, rooting, symmetric- and asymmetric tonic neck reflex. How do these involuntary reflexes turn into voluntary movement? Students experienced how hard it can be to change behavioural patterns in a reflex integration exercise, switching between inhibition of different parts of the ATNR patterns and adding them again. Interestingly, even though they knew the exercise was set up to 'overload' the students, they noticed frustration and being disappointed with themselves, thinking: "I should be able to do this.". Becoming aware of their way of coping with this frustration, they also found ways to continue to move and noticed improvement in a short period of practice.

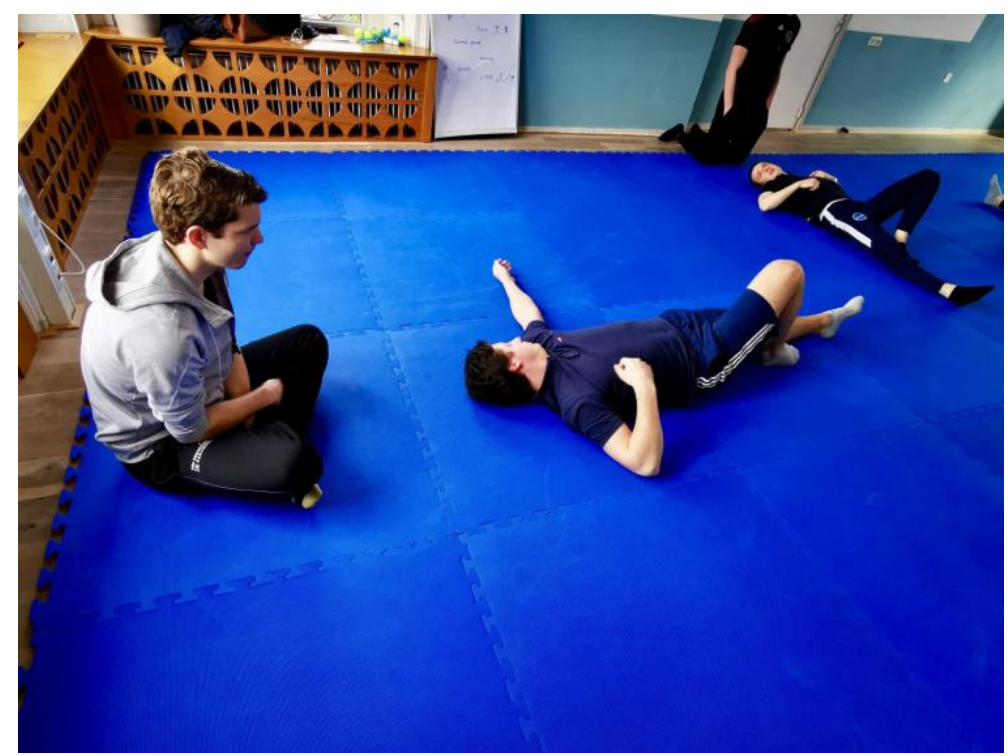

ATNR Reflex Integration Exercise. How do you cope with frustration in your learning process?

After a round of observations, the students were challenged to find a movement solution within different constraints. Laying on the floor, they had to move from back to stomach and back while keeping their hands and feet on - and later also off - the floor 
throughout the whole transition. Various solutions were found, while one student remarked: "I notice that I tend to continue with the same solution once I've found one - whereas that may not be the only, let alone most efficient solution".

Finally, after puzzling how to organise the limbs in order to roll over, we switched to a dyadic game in which movement solutions emerged from following a target. While one was holding the ball on the rope, the other had to follow the ball, first with the eyes (being able to see the ball), later with various body parts such as the finger, foot, elbow, ect. The challenge for the person leading the ball was to invite movement behaviour of their partner, essentially creating possibilities for action (affordances) for their partner. By locating the ball in a position in space at the verge of what was possible for the person following, he or she was challenged to explore and find new movement solutions, e.g., by reorganising the body by moving the upper body backwards so that the foot may stretch further forwards. Students observed that 'the body will organise itself', without having to think about where to 'place' various parts of the body. Bound by the dynamics of the body and the constraints of the environment, the organisation of the body converts towards the target.

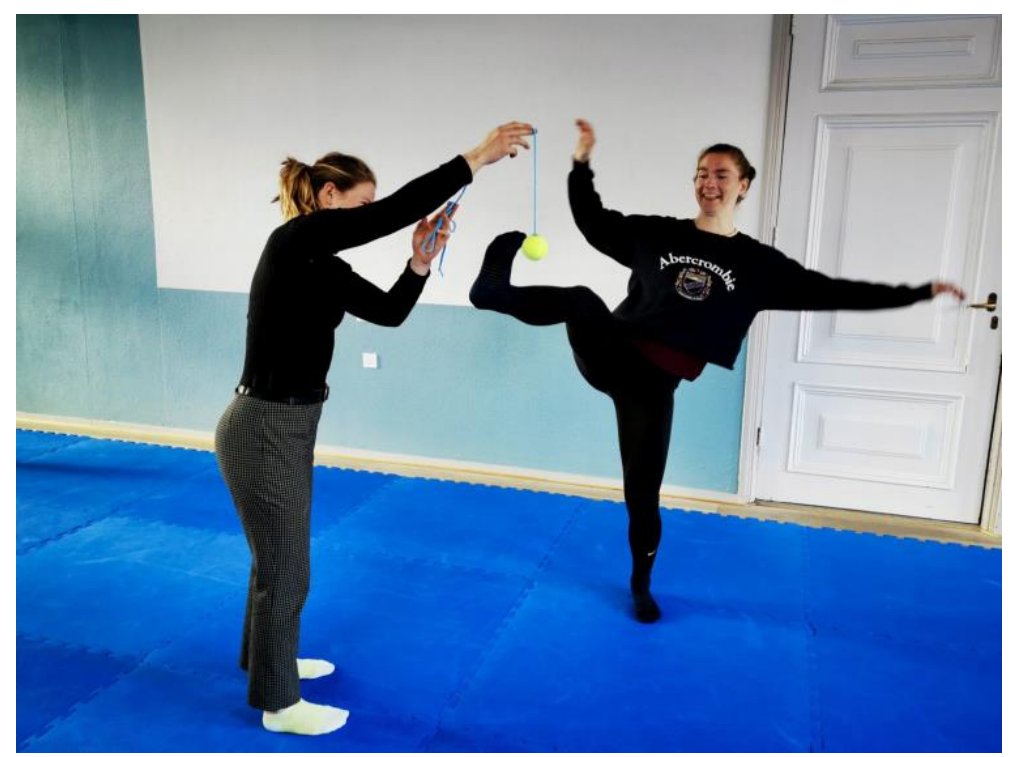

Challenging new movement solutions.

The movement workshop was a novel experience for the students (and educational experiment for me). One of the challenges teaching this workshop was to create a learning environment safe enough to invite exploration and play - especially given that exploration through movement and reflection upon body sensations are not within to the social norms of a 'normal' educational setting. By explaining the concepts and exercises while physically moving myself, showing the students what I meant, I made it the norm to move. Sharing my own experiences with these exercises during the rounds of reflection opened up the discussion for students to share theirs. In the end, I asked the students which exercise had made the most impact to them. The students indicated that they were taken by the ATNR and proprioceptive exercises - which were the exercises that confronted them with what they thought they should be able, but were not (yet) able to do. Together with their laughter during the ATNR and rolling exercises, this was a good sign: the best way to learn is through play, and in order to play, it has to be okay to succeed and fail. 


\section{Movement Workshop}

I greatly enjoyed teaching this workshop. Moving together on the mat and being jointly engaged in movement exploration seemed to close the distance between teacher and students. The interaction that opened up from that, sharing their experiences in the group and asking about mine, discussing the implications of their experiences for theory and questioning methods to research concepts such as reflexes, were what I had hoped for.

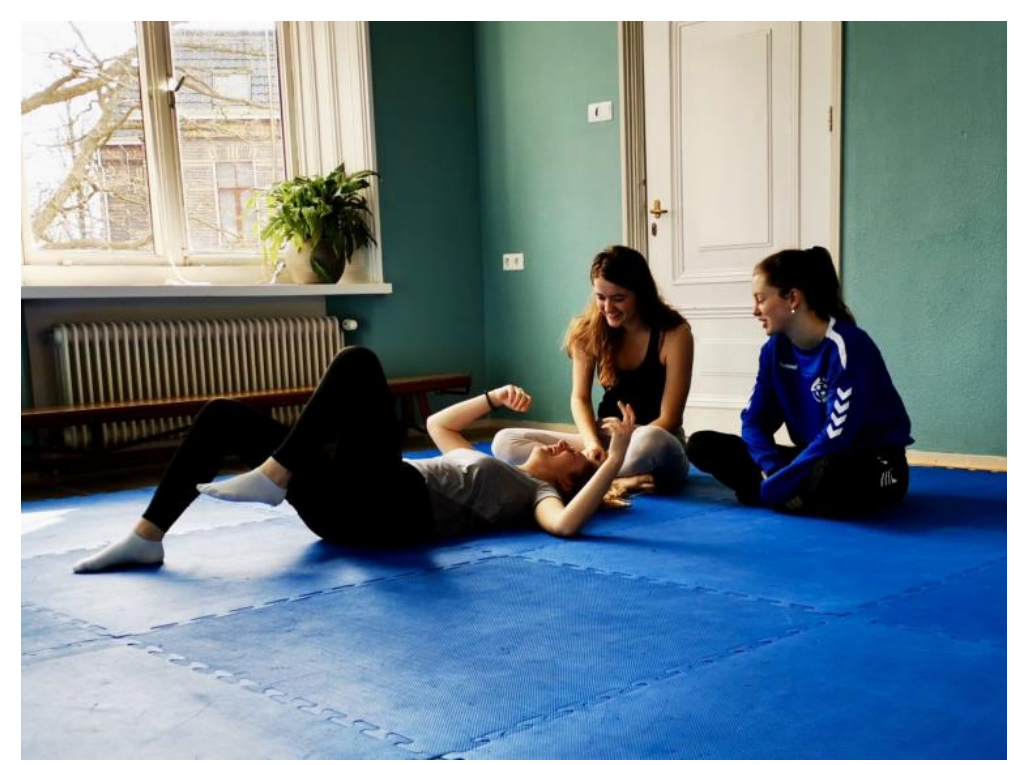

Having fun during the ATNR-exercises. 